\title{
Placement of lumen-apposing metal stents to drain pseudocysts and walled-off pancreatic necrosis can be safely performed on an outpatient basis: A multicenter study
}

Douglas G. Adler, Janak Shah ${ }^{1}$, Jose Nieto², Kenneth Binmoeller ${ }^{1}$, Yasser Bhat ${ }^{1}$, Linda Jo Taylor, Ali A. Siddiqui Division of Gastroenterology and Hepatology, University of Utah Hospital, Salt Lake City, UT, 'Division of Gastroenterology, California Pacific Medical Center, San Francisco, CA, ${ }^{2}$ Department of Gastroenterology, Borland-Groover Clinic, Jacksonville, FL, ${ }^{3}$ Division of Gastroenterology and Hepatology, Thomas Jefferson University Hospital, Philadelphia, PA, USA

\begin{abstract}
Backgrounds and Objectives: No study on the use of lumen-apposing fully covered self-expanding metal stent (LAMS) to drain pancreatic fluid collections (PFCs) has evaluated outcomes of patients in the outpatient setting. The objective of this multicenter study was to evaluate the clinical outcomes, success rate, and adverse events of the LAMS for endoscopic ultrasound (EUS)-guided transmural drainage of patients with symptomatic PFCs on an inpatient versus an outpatient basis. Methods: This was a multicenter, retrospective study conducted at 4 tertiary care centers. Results: We identified eighty patients with PFCs in whom EUS-guided transmural drainage using the LAMS was performed. The mean age of the patients was 53.1 years old. Mean size of the PFC was $11.8 \pm 5.1 \mathrm{~cm}$. A total of 33 patients had PFCs drained in an outpatient setting while 47 patients underwent PFC drainage as inpatients. The overall technical success (ability to access and drain a PFC by placement of transmural stents) was $98.7 \%$ (79 patients). There was no statistically significant difference in the technical success rate between the inpatient and outpatient groups (100\% vs. $98 \%$, respectively, $P=1)$. There was no significant difference in resolution of PFCs in the inpatient and outpatient groups $(91 \% v s .87 \%$ respectively; $P=1)$. The number of procedures required for PFC resolution was significantly lower in the inpatient group as compared to the outpatients ( 2.3 vs. 3.1 respectively, $P=0.025$ ). Procedure-related adverse events were significantly lower in the inpatient group compared to the outpatient group $(P<0.01)$. There was no significant difference in the 2 groups in terms of development of adverse events requiring endoscopic reintervention within 30 days of initial stent placement $(P=0.69)$. Conclusion: This study shows that LAMS placement for PFCs can be performed safely on an outpatient basis with overall technical and clinical outcomes that are comparable to those seen in inpatients.
\end{abstract}

Key words: Acute pancreatitis, endosonography, pancreatic fluid collections

\begin{tabular}{|l|l|}
\hline \multicolumn{2}{|c|}{ Access this article online } \\
\hline Quick Response Code: & Website: \\
\hline
\end{tabular}

This is an open access journal, and articles are distributed under the terms of the Creative Commons Attribution-NonCommercialShareAlike 4.0 License, which allows others to remix, tweak, and build upon the work non-commercially, as long as appropriate credit is given and the new creations are licensed under the identical terms.

For reprints contact: reprints@medknow.com

How to cite this article: Adler DG, Shah J, Nieto J, Binmoeller K, Bhat $\mathrm{Y}$, Taylor LJ, et al. Placement of lumen-apposing metal stents to drain pseudocysts and walled-off pancreatic necrosis can be safely performed on an outpatient basis: A multicenter study. Endosc Ultrasound 2019;8:36-42.

Address for correspondence

Dr. Ali A. Siddiqui, Thomas Jefferson University, 132 S. 10 $0^{\text {th }}$ Street, Main Building, Philadelphia, PA 19107, USA.

E-mail: asiddiqu2004@gmail.com

Received: 2016-12-20; Accepted: 2017-04-22; Published online: 2018-05-17 
Adler, et al:: Outpatient endoscopic therapy of pancreatic fluid collections

\section{INTRODUCTION}

Pancreatic fluid collections (PFCs) are commonly encountered clinical entities and are thought to arise due to pancreatic duct damage in patients with acute or chronic pancreatitis, pancreatic trauma, iatrogenic injuries (i.e., surgery), and in patients with a disrupted pancreatic duct. ${ }^{[1,2]}$ PFCs are generally divided into two groups: pancreatic pseudocysts (PPs) and walled-off necrosis (WON). PPs are defined as of fluid collections in the peripancreatic tissues that are surrounded by a well-defined wall and contain no solid material. WONs are defined as consisting of necrotic tissue contained within a wall of reactive tissue. ${ }^{[1]}$ Most PFCs are small and asymptomatic and many resolve spontaneously. Nonetheless, some PFCs become symptomatic usually due to a large size that produces when they increase in size or become infected, leading to symptoms including abdominal pain, early satiety, gastric outlet obstruction, biliary obstruction with concomitant jaundice, and sepsis. ${ }^{[3]}$

The current management options for symptomatic PFCs include endoscopic, surgical, and percutaneous drainage. ${ }^{[4-6]}$ The surgical approach is most invasive and carries a high mortality and morbidity. PFCs drained through the percutaneous approach are at risk of fistula formation, cyst recurrence, and infections. ${ }^{[7]}$

In the last decade, endosonography-guided drainage of PFCs through the placement of transmural stents has become the procedure of choice in many tertiary care centers, often viewed as the first-line therapy instead of surgical or interventional radiology approaches. Recently, a novel "saddle-shaped" lumen-apposing fully covered self-expanding metal stent (LAMS) with both proximal and distal anchor flanges has been demonstrated to be both safe and effective for endoscopic transmural drainage of PPs and WONs and is often used in place of a covered biliary metal stent or double-pigtail stents. ${ }^{[8-11]}$

To date, no study on the use of LAMS to drain PFCs has evaluated the role and outcomes of patients in the outpatient setting. It is unknown if LAMS placement warrants inpatient admission at the time of placement or is safe to be performed in outpatients. Other high-risk endoscopic procedures, including ERCP, percutaneous endoscopic gastrostomy, and interventional endoscopic ultrasound (EUS) procedures, are routinely performed in the outpatient setting. The objective of this large multicenter study was to evaluate the overall clinical outcomes, success rate, and adverse events of the LAMS for EUS-guided transmural drainage of patients with symptomatic PFCs in the United States, in those undergoing the procedure on an inpatient versus an outpatient basis.

\section{METHODS}

This was a multicenter, retrospective study conducted at 4 tertiary care centers. The study was approved by the Institutional Review Boards in all centers. The study concept, hypothesis, and design were investigator initiated and no financial support was received.

The endoscopy database at Thomas Jefferson University Hospital, University of Utah Hospitals and Clinics, Borland-Groover Clinic and California Pacific Medical Center was queried for all patients who had undergone EUS-guided drainage of PFCs (i.e., PP and WON) using the LAMS between February 2012 and June 2016. Only patients with a 3-month or greater follow-up were included in the study. WON consisted of a mature, encapsulated collection of pancreatic, and/or peripancreatic necrotic tissue contained within an enhancing wall of reactive tissue. PP was defined as an encapsulated collection of fluid with a well-defined inflammatory wall usually outside the pancreas with minimal or no necrosis (as per the Revised Atlanta Classification).$^{[1]}$

Inpatients were already admitted at the time of the procedure. Outpatients were patients who came in as outpatients to the endoscopy suite and in whom there were no plans to admit unless a complication occurred. Patients were hospitalized preprocedure if they had intractable pain, evidence of fevers, or inability to take oral intake or other symptoms of concern. Patients were admitted if they developed a complication from their endoscopy. PFCs were characterized by a variety of techniques including magnetic resonance imaging or computed tomography (CT) as well as EUS on the day of their initial drainage procedure. Indications for drainage of PFCs were as follows: (1) signs or symptoms of infection in the PFC, (2) abdominal pain felt to be attributable to the PFC, (3) gastric outlet or biliary obstruction due to extrinsic compression through the PFC, (4) ongoing systemic illness, anorexia, nausea, vomiting, and weight loss, or (5) rapidly enlarging PFCs. ${ }^{[12]}$ Patients with interposed vessels or significant varices, cystic neoplasms, 
coagulopathy (international normalized ratio $>1.6$ ), and significant thrombocytopenia (platelets $<50,000 / \mathrm{mm}^{3}$ ), or cyst imaging showing that the PFC wall was not in proximity $(>1 \mathrm{~cm})$ to the gastric or duodenal lumen were excluded from the study. Data were obtained from inpatient and outpatient hospital records to collect procedural details and overall clinical course of the patient. In addition, records from hospitals in our region are available through our electronic medical record to facilitate data capture from events occurring beyond the site of endoscopic intervention.

\section{Description of the lumen-apposing fully covered} self-expanding metal stent

The LAMS (AXIOS ${ }^{\mathrm{TM}}$; Boston Scientific, Natick MA) utilized in this study is a saddle-shaped, braided flexible stent made of nitinol that is fully covered with a silicon membrane. The stent is manufactured with bilateral double-walled anchoring flanges designed to hold the stomach or duodenal wall in close apposition to PFC wall. ${ }^{[13]}$ The stent is $10 \mathrm{~mm}$ in length and is available in 10 or $15 \mathrm{~mm}$ diameter sizes.

\section{Techniques}

All patients underwent procedures by endoscopists with $>5$ years of active EUS practice (D.G.A., A.A.S., J.N., J.N.S., K.F.B., Y.M.B.). All endoscopists have prior experience with PFC drainage through traditional techniques. PFC drainage was performed in all cases through a therapeutic linear array echoendoscope (GFUCT180; Olympus, USA). All procedures were performed under general anesthesia and all patients were administered broad-spectrum antibiotics during and after the procedure to decrease the risk of infection. A careful EUS examination before LAMS placement was used to determine the optimal puncture site of the cyst in all patients. Color Doppler was used to exclude interposed vessels at the puncture site 19-gauge EUS fine-needle aspiration needles were used to perform the initial puncture into the cyst under real-time imaging. The cyst contents were then aspirated for visual inspection (e.g., viscosity, debris, pus) and sent for fluid analysis (e.g., tumor markers, cytology, culture) if felt to be warranted the discretion of the endoscopist. A 0.035 " guidewire was then inserted through the needle and allowed to coil several times into the cyst cavity under EUS and fluoroscopic guidance. The needle was then withdrawn over the wire. Cystgastrostomy tract dilation was performed with either an 8F-10F Soehendra dilator (Cook Medical, Winston-Salem NC, USA) or a $6 \mathrm{~mm}$ or $8 \mathrm{~mm}$ wire-guided balloon (Hurricane, Boston Scientific, Natick MA, USA) based on the preference of the endoscopist. After the cystgastrostomy was dilated, the LAMS delivery catheter was advanced over the wire and into the cyst cavity. Of note, the LAMS device used in this study was the so-called "Cold-Axios" that did not have a diathermic tip on the catheter. Once the LAMS catheter was in good position in the PFC, the distal half of the stent was deployed under EUS guidance with or without fluoroscopic guidance at the discretion of the endoscopist. Deployment of the proximal flange of the stent was then performed under endoscopic guidance. The selection of stent diameter was at the discretion of the endoscopist; although, in general, if the PFC was felt to contain significant debris, a $15 \mathrm{~mm}$ LAMS was placed to facilitate the future necrosectomy procedures. The deployed stent lumen was then dilated up to 10 or $15 \mathrm{~mm}$ with a controlled radial expansion balloon (Boston Scientific) to allow for optimal stent luminal expansion if felt to be indicated by the endoscopist.

In patients with WON, subsequent endoscopic necrosectomy procedures were performed using a standard upper endoscope advanced through the LAMS at times dictated by the preference of the endoscopist, usually every 3-7 days until complete resolution of the necrotic cavity as confirmed endoscopically and/or by results obtained through cross-sectional imaging.

Any complication occurring or after during the procedure including perforation, bleeding, infection, development of pancreatitis or worsening of existing pancreatitis, hypotension, or respiratory distress was carefully documented. The electronic medical records of hospital admissions, emergency room visits, and/or clinic visits were also compiled in an effort to identify any delayed complication (up to 30 days after procedure).

\section{Patients' follow-up}

All patients underwent contrast-enhanced CT of the abdomen at 4-8 weeks after LAMS placement followed in an outpatient clinic visit. LAMS removal was performed when complete cyst decompression was achieved, i.e., the PFC had completed resolved without any residual intracystic solid or fluid component remaining.

\section{Outcomes measures}

Technical success was defined as the ability to place and 
Adler, et al:: Outpatient endoscopic therapy of pancreatic fluid collections

deploy a LAMS stent transmurally without difficulty. Successful drainage of the PFC based on endoscopy or cross-sectional imaging results 1 month after the therapy with at least a $50 \%$ reduction in PFC size and resolution of clinical symptoms as documented in clinic follow-up notes, respectively. ${ }^{[14]}$

Immediate procedure-related adverse events were defined as those that occurred within 1 week after the procedure. Reinterventions included the need to replace or remove migrated LAMS, repeat PFC drainage due to LAMS stent occlusion, cyst cavity infection with inadequate drainage through the LAMS, enlarging cyst size despite LAMS placement, or worsening of clinical symptoms due to the PFC.

The primary outcome of this study was to evaluate the overall long-term success and adverse event rate of LAMS usage in inpatients versus outpatients. Patients were considered inpatients if they were either inpatients at the time of PFC placement or admitted to the hospital after LAMS placement. Patients were considered outpatients if they came from home for their LAMS placement and returned home the same day without being admitted to the hospital at all. Outpatients were carefully monitored for any adverse event that would require inpatient admission after being discharged to home.

Long-term success was defined as complete resolution of the pseudocyst and resolution of the patient's symptoms without need for reintervention at 3 months following initial treatment as demonstrated by ambulatory clinic follow-up and cross-sectional imaging. Secondary outcomes evaluate included adverse events, number of endoscopic reinterventions, and PFC recurrence rates after the stent removal.

\section{Statistical analysis}

This was a retrospective cohort study. We divided the subjects into 2 groups according to the setting of the PFC drainage 1) outpatient and 2) inpatient. Outcomes between the groups were compared using the Student's $\mathrm{t}$ test for continuous variables and the Chi-square test for categorical variables. Generalized linear modeling (GLM) were performed on categorical and continuous variables, respectively, in order to assess any difference in outcomes in the outpatient versus inpatient setting. Statistical significance was determined a priori at $P \leq$ 0.05 .

\section{RESULTS}

\section{Patient demographics and pancreatic fuid collection characteristics}

From February 2012 to June 2016, we identified 80 patients with PFCs in whom EUS-guided transmural drainage using the LAMS was performed. All PFCs developed after the episodes of acute pancreatitis. The mean age of the patients was $53.1 \pm 15$ years and $40 \%$ were female. The etiology of pancreatitis was gallstone (49\%), alcohol (30\%), idiopathic $(8 \%)$, drug-induced $(2 \%)$, autoimmune $(1 \%)$, and hypertriglyceridemia $(10 \%)$. The clinical presentation in patients that required drainage included abdominal pain, nausea and vomiting, anorexia and early satiety, and biliary obstruction [Table 1].

PFCs were located in the pancreatic head (5\%), pancreatic body and tail (84\%), and involved the pancreatic head/body/tail (11\%). Mean size of the $\mathrm{PFC}$ was $11.8 \pm 5.1 \mathrm{~cm}$ in the long axis (range, $4.8-25 \mathrm{~cm})$.

Of the eighty patients, 12 presented with PP and 68 patients had WONs. There was no significant difference in sex, etiology, or cyst size between patients with PP and WON. Patients with WON were younger (51.7 vs. 61 years respectively, $P=0.04$ ) and were predominantly white $(82.3 \%$ vs. $50 \%$ respectively, $P=0.02)$ when compared to those with PP. Twenty percent of patients had failed prior endoscopic or interventional radiology intervention before our endoscopist intervention.

\section{Inpatients and outpatients that underwent pancreatic fluid collection drainage}

There were 33 patients whose PFC cavity was drained in an outpatient setting while 47 patients underwent PFC drainage using the LAMS as inpatients. Transgastric drainage was performed in 74 patients $(92.5 \%), 5$ patients $(6.25 \%)$ had transduodenal drainage, and $1(1.25 \%)$ patients had multiport stent drainage/debridement. Seventy-two patients had a $15 \mathrm{~mm}$ wide $\mathrm{x} 10 \mathrm{~mm}$ long LAMS placed and 8 had a $10 \mathrm{~mm} \times 10 \mathrm{~mm}$ diameter LAMS placed. The median duration that the stents remained implanted was 1 month (range, 1-6 months). Nine patients had a concomitant ERP with transpapillary pancreatic stent placement for a concomitant pancreatic duct leak contributing to WONs as seen on MRCP. Twenty-two patients had placement of an $8 \mathrm{Fr}$ nasocystic catheter 
due to the presence of large amount of solid debris inside the WON cavity and nasocystic lavage was performed with bolus perfusions of saline for 1-2 days after initial LAMS placement [Table 2].

The overall technical success (ability to access and drain a PFC by the placement of transmural stents) was 98.7\% (79 patients). There was no statistically significant difference in the technical success rate between the inpatient and outpatient groups (100\% vs. $98 \%$ respectively, $P=1$ ). Unsuccessful placement of the LAMS in 1 patient in the outpatient group was as a result of stent maldeployement.

\section{Long-term follow-up (6 months)}

On 6-month follow-up, overall clinical success with successful eradication of the PFC was achieved in $72 / 80(90 \%)$ patients. There was no difference in the percentage of inpatients $(84.8 \%)$ and outpatients $(85.1 \%)$ who were found to have WON. Similarly, there was no difference in the number of patients with WON in whom elective direct endoscopic necrosectomy (DEN) was performed when comparing the inpatient and outpatient groups $(78.8 \%$ vs. $78.7 \%$, respectively; $P=1$ ). There was no significant difference in complete resolution of PFCs in the inpatient and outpatient groups ( $91 \%$ vs. $87 \%$, respectively; $P=1$ ). The mean number of procedures required for PFC resolution after initial stent placement was significantly lower in the inpatient group as compared to the outpatients (2.3 vs. 3.1, respectively, $P=0.025$ ).

There was no significant difference in resolution of WON between patients with or without nasocystic tube irrigation placed at the time of initial procedure (93.2\% vs. $87.4 \%$, respectively; $P=0.26)$. Similarly, there was no significant difference in resolution of the WON between patient with or without transpapillary pancreatic stent placement at the time of initial procedure $(90 \%$ vs. $88.7 \% ; P=1)$. No recurrence of WON or a pseudocyst was seen after the removal of the stents in any of the groups. These findings are summarized in Table 3.

\section{Procedure-related adverse events}

There was one procedure-related adverse event in the inpatient group; this was significant hemorrhage due to inadvertent puncture of an artery, which was successfully treated with coil embolization by interventional radiology [Table 2].
There were eight procedure-related adverse events in the outpatient group: 3 perforations, 6 episodes of clinically significant bleeding at the cystenterostomy/ stent site, 1 suprainfection, and 3 episodes of self-limited bleeding at the stent site after the procedure that resolved with local injection of epinephrine. One 63 year old male patient who developed a perforation after stent maldeployement had the perforation closed using an over-the-scope clip; he then had placement of a fully covered biliary metal stent through a new puncture site to drain the WON; this patient did well and had complete resolution of his WON. Two patients developed pneumoperitoneum after failed transmural drainage/ debridement due to LAMS maldeployement. The LAMS were removed. The operators then could not successfully advance the guidewire into the WON cavity. The guidewire was repeatedly advanced into the abdominal cavity despite multiple attempts; therefore, another stent could not be placed safely to drain the pseudocyst. These patients were sent to surgery and had primary repair of the gastric perforation and underwent cystgastrostomy.

Procedure-related adverse events were significantly lower in the inpatient group compared to the outpatient group $(P<0.01)$. There was no procedure-related mortality in either group.

Late adverse events after initial stent placement In the inpatient group, 3/33 (9\%) patients required reintervention due to the following reasons: 1 patient had occlusion of the LAMS as a result of necrotic debris and 2 patients had infection of the PFC cavity requiring endoscopic debridement and irrigation.

The need for endoscopic reintervention in the outpatient group was seen in $3 / 47(6 \%)$ of cases due to the following reasons: one patient had occlusion of the LAMS as a result of necrotic debris and 2 patients had infection of the PFC cavity requiring endoscopic debridement and irrigation. There was no significant difference in the 2 groups in terms of development of adverse events requiring endoscopic reintervention within 30 days of initial stent placement $(P=0.69)$. Patients who had secondary infection of the PFC as a result of occluded stents were all successfully managed by endoscopic replacement of stents and placement of a nasocystic drain.

\section{DISCUSSION}

The use of LAMS to endoscopically drain and debride PFCs including PP and WON is undergoing 
Adler, et al:: Outpatient endoscopic therapy of pancreatic fluid collections

a period of exponential growth with this technology rapidly disseminating into widespread clinical practice. Historically, PFC drainage and LAMS placement have often perceived as a procedure that frequently warrants inpatient hospitalization. This study demonstrates that the placement of LAMS in patients with PFCs can be safely performed on an outpatient basis and does not automatically warrant inpatient hospitalization.

This study included 80 patients, the vast majority of whom had WON. As such, this represents a cohort of, overall, significantly ill patients. The overall technical success in our study was very high (98.7\%) and was similar to, or better than, that seen in other studies of the use of LAMS to drain PFCs. ${ }^{[15-17]}$ There was no statistically significant difference in the technical success rate between the inpatient and outpatient groups. The proportion of patients with WON was the same in our inpatient and outpatient subgroups as was the percentage of patients in both groups who required DEN, further demonstrating that outpatient LAMS placement and debridement can be performed in patients with sequelae of severe acute pancreatitis.

Our study showed no significant difference in the rate of complete resolution of PFCs in the inpatient and outpatient groups $(91 \%$ vs. $87 \%)$. The number of procedures required for $\mathrm{PFC}$ resolution after the initial stent placement was statistically significantly lower in the inpatient group as compared to the outpatient although this essentially amounted to only one more procedure in the outpatient group (approximately 2 vs. 3, respectively). The reason for this difference in total number of procedure required is unclear at this time.

There were more adverse events related to the LAMS placement itself in the outpatient group, but there was no significant difference in the 2 groups in terms of development of adverse events requiring endoscopic reintervention within 30 days of initial stent placement. It is unclear why there were more adverse events during the LAMS placement in the outpatient group although patients who are referred for planned outpatient LAMS procedures can always be admitted afterward for subsequent observation and/or care as needed. The fact that the rate of delayed adverse events was the same between the two groups argues that in patients who undergo an uneventful LAMS procedure further demonstrates that these patients can be safely discharged without inordinate fear of poor outcomes.
Table 1. Patient demographics and pancreatic fluid collection characteristics

\begin{tabular}{lc}
\hline & Patients $(n=80)$ \\
\hline Gender (\%) & 40 \\
Female & 60 \\
Male & 53.1 \\
Mean age (years) & \\
Race (\%) & 78 \\
White & 6 \\
Black & 10 \\
Hispanic & 6 \\
Other & \\
Pancreatitis etiology (\%) & 49 \\
Gallstone & 30 \\
Alcohol & 8 \\
Idiopathic & 0 \\
Trauma & 1 \\
Autoimmune & 10 \\
High triglycerides & 2 \\
Drug related & 118 \\
Mean WON long axis measurement (mm) & \\
Site of WON (\%) & \\
Pancreatic head & \\
Pancreatic body/tail & 11 \\
Pancreatic head/body/tail & \\
\hline WON: Walled-off necrosis & \\
\hline
\end{tabular}

WON: Walled-off necrosis

Table 2. Procedural characteristics and adverse events

\begin{tabular}{lcc}
\hline & $\begin{array}{c}\text { Out patients } \\
(n=33)\end{array}$ & $\begin{array}{c}\text { In patients } \\
(n=47)\end{array}$ \\
\hline Site of cyst-enterostomy (\%) & 94 & 91 \\
$\quad$ Stomach & 6 & 9 \\
$\quad$ Duodenal bulb & 98 & 100 \\
Procedural technical success (\%) & & \\
Procedural adverse events & 10 & 3 \\
$\quad$ Bleeding & 2 & 0 \\
Suprainfection & 4 & 0 \\
$\quad$ Perforation & & \\
\hline
\end{tabular}

Table 3. Results of endoscopic ultrasound-guided drainage/debridement of pancreatic fluid collections

\begin{tabular}{lcc}
\hline & $\begin{array}{c}\text { Outpatients } \\
(n=33)\end{array}$ & $\begin{array}{c}\text { Inpatients } \\
(n=47)\end{array}$ \\
\hline $\begin{array}{l}\text { Mean number of endoscopic } \\
\text { sessions for PFC resolution }\end{array}$ & 3.1 & 2.3 \\
$\begin{array}{l}\text { Success rate for endoscopic } \\
\text { drainage of WON (\%) }\end{array}$ & 87 & 91 \\
$\begin{array}{l}\text { Patients that required } \\
\text { radiological and/or surgery } \\
\text { for final PFC therapy (\%) }\end{array}$ & 8.5 & 6 \\
$\begin{array}{l}\text { Recurrence of PFC } \\
\text { after endoscopic stent } \\
\text { removal (\%) }\end{array}$ & 0 & 0 \\
\hline PFC: Pancratic filcolection WON: Walled-off necrosis &
\end{tabular}

PFC: Pancreatic fluid collection, WON: Walled-off necrosis

Our study shows that as experience grows with interventional EUS procedures in general, and PFC 
Adler, et al:: Outpatient endoscopic therapy of pancreatic fluid collections

drainage specifically, our comfort level with performing these procedures in outpatients can grow as well. A similar evolution occurred in the world of ERCP approximately 2 decades ago. Previously, ERCP-based maneuvers such as sphincterotomy, stone extraction, and stent placement were felt to warrant subsequent inpatient admission and observation. Studies from the 1990s showed that these and other maneuvers could be safely performed on outpatients. ${ }^{[12-14,18]}$ Similar findings were seen when laparoscopic cholecystectomy transitioned from a purely inpatient procedure to a predominately outpatient procedure. ${ }^{[19-21]}$

Overall, this study shows that LAMS placement for PFCs including both PP and WON can be performed safely on an outpatient basis with overall technical and clinical outcomes that are comparable to those seen in inpatients. Patients with a planned outpatient LAMS procedure who develop an adverse event during the procedure can always be admitted for inpatient care afterward. Stable outpatients who tolerate LAMS placement without difficulty can be safely discharged home on the same day as their procedure.

\section{CONCLUSION}

This study shows that LAMS placement for PFCs can be performed safely on an outpatient basis with overall technical and clinical outcomes that are comparable to those seen in inpatients.

\section{Financial support and sponsorship}

This study was funded entirely through existing intramural funds and salary support.

\section{Conflicts of interest}

Douglas G. Adler is a speaker and consultant for Boston Scientific Janak Shah is a consultant for Boston Scientific Jose Nieto is a speaker and consultant for Boston Scientific Kenneth Binmoeller is a consultant for Boston Scientific and inventor of the LAMS Yasser Bhat has no conflicts of interest Linda Jo Taylor has no conflicts of interest Ali A. Siddiqui is a consultant for Boston Scientific.

\section{REFERENCES}

1. Banks PA, Bollen TL, Dervenis C, et al. Classification of acute pancreatitis - 2012: Revision of the Atlanta classification and definitions by international consensus. Gut 2013;62:102-11.

2. Brun A, Agarwal N, Pitchumoni CS. Fluid collections in and around the pancreas in acute pancreatitis. J Clin Gastroenterol 2011;45:614-25.

3. Gurusamy KS, Pallari E, Hawkins N, et al. Management strategies for pancreatic pseudocysts. Cochrane Database Syst Rev 2016;4:CD011392.

4. Tyberg A, Karia K, Gabr M, et al. Management of pancreatic fluid collections: A comprehensive review of the literature. World J Gastroenterol 2016;22:2256-70.

5. Vilmann AS, Menachery J, Tang SJ, et al. Endosonography guided management of pancreatic fluid collections. World J Gastroenterol 2015;21:11842-53.

6. Logue JA, Carter CR. Minimally invasive necrosectomy techniques in severe acute pancreatitis: Role of percutaneous necrosectomy and video-assisted retroperitoneal debridement. Gastroenterol Res Pract 2015;2015:693040.

7. Solanki R, Koganti SB, Bheerappa N, et al. Disconnected duct syndrome: Refractory inflammatory external pancreatic fistula following percutaneous drainage of an infected peripancreatic fluid collection. A case report and review of the literature. JOP 2011;12:177-80.

8. Lee HS, Chung MJ. Past, present, and future of gastrointestinal stents: New endoscopic ultrasonography-guided metal stents and future developments. Clin Endosc 2016;49:131-8.

9. Siddiqui AA, Adler DG, Nieto J, et al. EUS-guided drainage of peripancreatic fluid collections and necrosis by using a novel lumen-apposing stent: A large retrospective, multicenter U.S. experience (with videos). Gastrointest Endosc 2016;83:699-707.

10. Siddiqui AA, Kowalski TE, Loren DE, et al. Fully covered self-expanding metal stents versus lumen-apposing fully covered self-expanding metal stent versus plastic stents for endoscopic drainage of pancreatic walled-off necrosis: Clinical outcomes and success. Gastrointest Endosc 2017;85:758-65.

11. Walter D, Will U, Sanchez-Yague A, et al. A novel lumen-apposing metal stent for endoscopic ultrasound-guided drainage of pancreatic fluid collections: A prospective cohort study. Endoscopy 2015;47:63-7.

12. Elfant $A B$, Bourke MJ, Alhalel R, et al. A prospective study of the safety of endoscopic therapy for choledocholithiasis in an outpatient population. Am J Gastroenterol 1996;91:1499-502.

13. Bjorkman DJ, Van Dam J. Outpatient therapeutic ERCP: Cutting sphincters and cutting costs. Am J Gastroenterol 1996;91:1485-6.

14. Tham TC, Vandervoort J, Wong RC, et al. Therapeutic ERCP in outpatients. Gastrointest Endosc 1997;45:225-30.

15. Gornals JB, De la Serna-Higuera C, Sánchez-Yague A, et al. Endosonography-guided drainage of pancreatic fluid collections with a novel lumen-apposing stent. Surg Endosc 2013;27:1428-34.

16. Itoi $\mathrm{T}$, Binmoeller KF, Shah J, et al. Clinical evaluation of a novel lumen-apposing metal stent for endosonography-guided pancreatic pseudocyst and gallbladder drainage (with videos). Gastrointest Endosc 2012;75:870-6.

17. Rinninella E, Kunda R, Dollhopf M, et al. EUS-guided drainage of pancreatic fluid collections using a novel lumen-apposing metal stent on an electrocautery-enhanced delivery system: A large retrospective study (with video). Gastrointest Endosc 2015;82:1039-46.

18. Mehta SN, Pavone E, Barkun AN. Outpatient therapeutic ERCP: A series of 262 consecutive cases. Gastrointest Endosc 1996;44:443-9.

19. Arregui ME, Davis CJ, Arkush A, et al. In selected patients outpatient laparoscopic cholecystectomy is safe and significantly reduces hospitalization charges. Surg Laparosc Endosc 1991;1:240-5.

20. Ji W, Ding K, Li LT, et al. Outpatient versus inpatient laparoscopic cholecystectomy: A single center clinical analysis. Hepatobiliary Pancreat Dis Int 2010;9:60-4.

21. Lillemoe KD, Lin JW, Talamini MA, et al. Laparoscopic cholecystectomy as a "true" outpatient procedure: Initial experience in 130 consecutive patients. J Gastrointest Surg 1999;3:44-9. 